\title{
Integrative Vectors for Gene Deletion and Replacement
}

\author{
MARIAN SEKTAS*, MAGDALENA GREGOROWICZ, MAGDALENA KUCHARSKA \\ and EWA JODELKO
}

Department of Microbiology University of Gdańsk, Gdańsk, Poland

Received 3 July 2012, revised 15 November 2012, accepted 15 November 2012

\author{
Abstract
}

An improved method for gene deletion or replacement in Escherichia coli was developed. It employs a set of integrative vectors and two helper plasmids, as a temporary source of RecA and Flp activities. The integrative vectors combine several useful features including three different selection markers placed between two parallel oriented Flp recombinase target (FRT) sites. Each marker is flanked by two MCSs, for cloning the chosen homologous fragments of DNA to gene targeting. The vectors contain two properly oriented $E$. coli Chi sites for recombination enhancement. When required, selection markers can be excised from the chromosome resulting in unmarked strains.

Key words: gene deletion; gene replacement, integrative rectors

All techniques for quick and precise modification of the bacterial chromosome to make it more useful with indispensable genes are very important for optimizing biotechnological processes. Genetic manipulations like gene replacement or gene knockout can be achieved by a variety of techniques (Sektas, 2000). Since Escherichia coli strains serve as the host organisms for many research and commercial applications (Feher et al., 2012), the need for specific host with modified genome still exists. To transfer a gene from the plasmid to the chromosome in homologous recombination-mediated reaction some methods use strains defective in polA (Gay, 1984) or temperature-sensitive replicons (Hamilton et al., 1989; Posfai et al., 1997, Martinez-Morales et al., 1999). The procedure may also rely on integrative vector devoid of origin of replication (Posfai et al., 1994, Sektas and Szybalski, 2001) or Rep function depletion (Wild et al., 1998). Another approach is to use linear DNA that contains a target gene flanked by homologous regions of chromosome to replace the specific locus. It requires a certain genetic background, e.g. $\operatorname{rec} B C$ (Jasin and Schimmel, 1984) or recD genotypes (Biek and Cohen, 1986; Cherepanov and Wackernagel, 1995), or a means of inhibition of ExoV activity (Datsenko and Wanner, 2000). Alternatively, the presence of properly oriented Chi sequences on the DNA substrates is required (Dabert and Smith, 1997; Karoui et al., 1999). Dabert and Smith (1997) reported strong stimulation of gene replacement in E. coli using relatively short DNA fragments with Chi sequences near each end. RecBCD multifunctional enzyme complex approaches Chi by its diffusion from a double-stranded end in orientation-dependent manner, from right of Chi sequence 5'-GCTGGTGG-3' (Smith, 2012). By continuing unwinding of the DNA, the RecBCD enzyme produces singlestranded DNA substrate for the RecA protein (Roca and Cox, 1997). Recombination between a linear DNA fragment and the circular chromosome requires an even number of exchanges to maintain circularity of the chromosome and hence viability of the cell.

Our integrating system is based on prevention of DNA substrate degradation in $\mathrm{RecBCD}{ }^{+}$bacteria and stimulation of recombinantion by supplying RecA from helper plasmid pRecAts $\left(A^{R}\right)$. The recA gene expression is driven by IPTG-dependent $P_{\text {lac }}$ promoter. Since this plasmid was constructed based on pSC101 temperature-sensitive replicon it can be easily cured by cultivation under non-permissive conditions (Posfai et al., 1997). To test our method for gene targeting in wild-type E. coli MM294 (Meselson and Yuan, 1968), we constructed three basic integrative vectors differing by the presence of resistance genes: $\mathrm{Cm}^{\mathrm{R}}, \mathrm{Km}^{\mathrm{R}}$ or $\mathrm{Tc}^{\mathrm{R}}$, respectively. The antibiotic resistance genes are flanked by two parallel oriented FRT sites allowing for excision in vivo under conditions of transient expression of FLP gene from a conditional suicidal vector $\mathrm{pFT}-\mathrm{Km}$

* Corresponding author: M. Sektas, Department of Microbiology University of Gdańsk, Wita Stwosza 59, 80-308 Gdańsk, Poland; e-mail: marian.sektas@biol.ug.edu.pl 


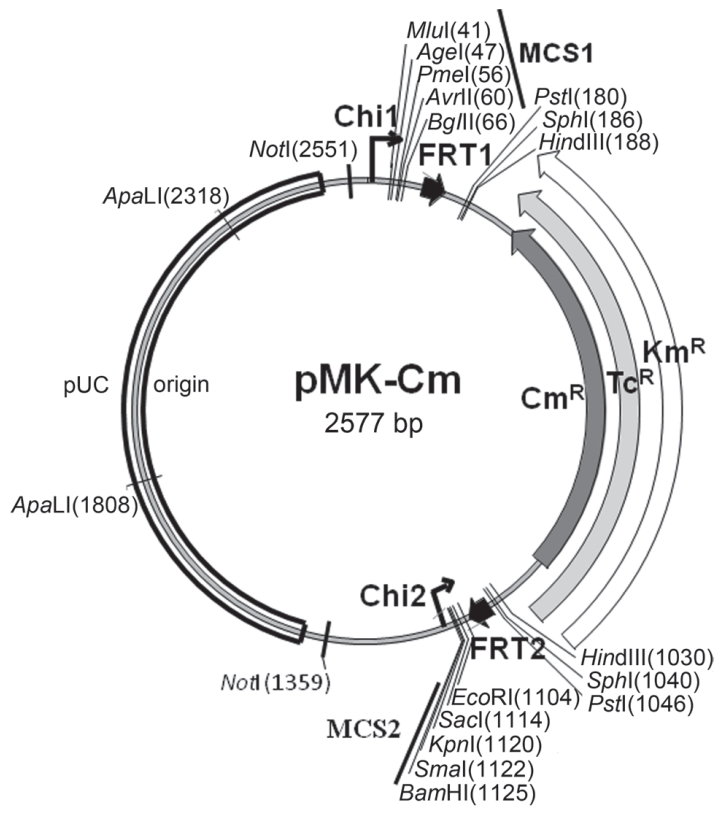

Fig. 1. Structural features of the pMK-family integrative vectors. Only key and unique restriction sites are marked.

Abbreviations: Chi, 5'-GCTGGTGG sequence; $\mathrm{Cm}^{\mathrm{R}}$, chloramphenicol resistance gene; FRT, 5'-GAAGTTCCTATTCTCTAGAAAGTATATAGGAACTTC sequence; $\mathrm{Km}^{\mathrm{R}}$, kanamycin resistance gene; $\mathrm{Tc}^{\mathrm{R}}$, tetracycline resistance gene.

(Posfai et al., 1997). Moreover, there are two polylinkers, MCS1 and MCS2, with five unique cloning sites each and two E.coli Chi sequences outside of MCSs. The presence of Chi sites on both ends of integration cassette in productive orientation may also stimulate gene replacement (Dabert et al., 1992). These vectors can be used in broad spectrum of hosts, both as a linear or non-replicating ori ${ }^{-}$circle. Fig. 1 shows all essential elements of pMK-vectors. The integrative plasmids were constructed in a series of steps starting with the pCANTS as a backbone (Posfai et al., 1994), which is a pBR322 derivative (Bolivar et al., 1977), possessing ori ${ }_{\mathrm{pBR}}$ as a cassette between two NotI restriction sites. To alter medium copy ori $i_{\mathrm{pBR} 322}$ to copy-up of ori $i_{\mathrm{pUC1}}, \mathrm{PCR}$ method was used. We designed two primers specific to pUC19 plasmid (Yanish-Perron et al., 1985), bearing NotI sites at either end (underlined): PUC3' (5'AAAGCGGCCGCAGGAAAGAACATGTGAGCA) complementary to the AflIII site (italic) [806 bp of pUC19]) and PUC5' (5'-AAATGCGGCCGCTAGGTGAAGATCCTTTTTG) close to the DraI site (1563 bp). 781 bp PCR-product was cut by NotI and inserted between NotI sites of pCANTS to give rise to pMS-Cm. Then, $430 \mathrm{bp}$ fragment of AflIII-NotI of pCANTS was inserted between the analogous sites of pMS-Cm (prepared by incomplete digestion of NotI), to extend the ori region to pas sequences (Bolivar et al, 1977). Thus obtained plasmid was named pMSS-Cm. To introduce E. coli Chi sequence (underlined) into the construct, two oligonucleotides were synthesized (Chil 5'-TCGACAGCTGCTGGTGGA-3' and Chi2 5'-AGCTTCCACCAGCAGCTG) to fit between SalI and HindIII of pMSS-Cm. Digestion with HindIII, DNA polymerase Klenow filling in and re-ligation allowed to alter HindIII site to NheI specific sequence.

To insert a cat gene (HincII fragment from pKRP10 vector, Reece and Phillips, 1995) between two parallel minimal FRT sites, plasmid pminFRTlac1.1 (originally derived from pMSlacZ.1, Sektas and Szybalski, 1998) was used. This plasmid was first prepared by BamHI and XhoI cutting followed by Klenow fragment of DNA I polymerase filling-in. Then, Chi site from pMSS-Cm was inserted between SalI and NheI sequences. Next, 41 bp SalI-XhoI fragment of MCS of pBend2 (Kim et al., 1989) was inserted into the SalI sites. Next, EcoRI-XhoI fragment of DNA (blunted by Klenow polymerase) containing the following set of genetic elements: FRT-cat-FRT-MCS1-Chi, was subcloned into pMSS-Cm between DraI and EcoRI sites. To broaden the set of unique restriction sites of MCS2, a polylinker was designed to introduce new AgeI, PmeI, AvrII sites between unique MluI and BglII (BglII primer 5'-GATCTCCTAGGTTTAAACACCGGTA and MluI primer 5'-CGCGTACCGGTGTTTAAACCTAGGA) and thus final plasmid pMK-Cm was created. Plasmids pMK-Tc and pMK-Km are identical to $\mathrm{pMK}-\mathrm{Cm}$ in all details but have genes conferring tetracycline or kanamycin resistance (Reece and Phillips, 1995), respectively (Fig. 1).

The integrative plasmids can recombine by a single or double crossover with homologous targeted sites, either as circular or linear form of DNA. The way of vector utilization is depended on whether one or two homologous fragments of DNA are to be cloned. All vectors contain removable pUC origin of replication, located between two NotI sites, giving the possibility of its deletion. A rapid construction of the ori-less ( $\Delta$ ori) "suicide" plasmid was carried out by using NotI and ApaLI digestions, followed by agarose-gel purification procedure. Upon introduction of the homologous DNA into E. coli MM294 by the standard transformation procedure, such $\Delta$ ori circle can integrate by a means of homologous recombination to the chromosomal target site. To test the insertion efficiency of linear form of DNA pMK-Cm vector was chosen, where truncated lacI gene (450 bp MluI-EcoRV gene fragment) was cloned into MCS1 site, and similarly, internal segment of lacZ (1716 Bsu36I-SacI gene fragment) was inserted into MCS2 site (pMK-CmlacIZ, Fig. 2B). Homologous replacement of the chromosomal $\mathrm{lac}^{+}$locus with these sequences should generate a $\mathrm{Lac}^{-} \mathrm{Cm}^{+}$phenotype. 
A

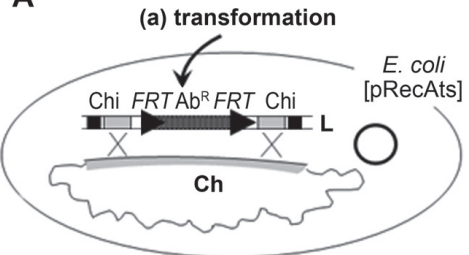

(b) $A b^{R}$ selection and

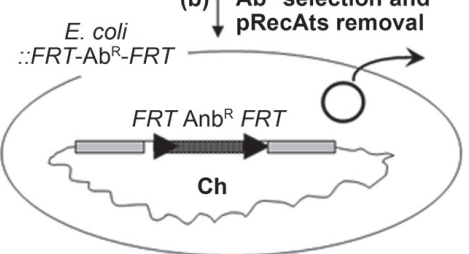

(c) $\mathrm{pFT}-\mathrm{Km}$ transformation FLP expression and removal

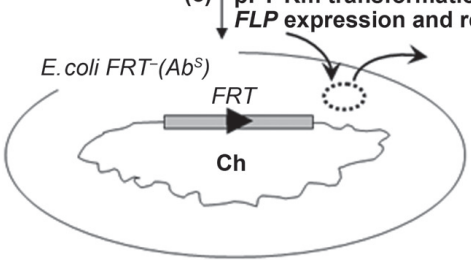

Fig. 2. Strategy and validation of unmarked gene disruption method

A. Schematic representation of the three step method: (a) transformation of integrative DNA, (b) selection of recombinants and pRecAts removal, and (c) marker excision and pFT-Km removal. Abbreviations: $\mathrm{Ab}^{\mathrm{R}}$ - antibiotic resistance, $\mathrm{Ab}^{\mathrm{S}}$ - antibiotic sensitivity, Ch - chromosome, L - linear dsDNA. B. Illustration of recombination-mediated chromosomal integration/removing of cat gene in lac locus of E.coli MM294. pMK-CmlacIZ integrative plasmid containing truncated lacI and $l a c Z$ genes was used along with two helper plasmids pRecAts $\left(\mathrm{Ap}^{\mathrm{R}}\right)$ and pFT-Km $\left(\mathrm{Km}^{\mathrm{R}}\right)$, to provide the RecA and Flp functions, respectively. C. PCR analysis of allelic exchange and subsequent marker removal corresponding to panel B. Total DNA from MM294 and its recombinant derivatives was isolated and subjected to PCR reactions. The PCR products were separated on $0.8 \%$ agarose gel. Lane 1 - product $\mathrm{A}$ of 1140 bp (MM294), lane 2, product C of 1500 bp (MM294lac::cat $\left[\mathrm{Cm}^{\mathrm{R}}\right]$ ), lane 3, DNA fragments from product $\mathrm{C}$ after HindIII digestion (840and $580 \mathrm{bp}$ ), lane 4 , product $\mathrm{D}$ of $570 \mathrm{bp}\left(\mathrm{MM} 294 \mathrm{lac} \Delta \mathrm{cat}\left[\mathrm{Cm}^{\mathrm{s}}\right]\right)$ ), lane 5 , negative control with Cat2 and LacI5 pair of primers (MM294), lane 6, product $\mathrm{B}$ of $680 \mathrm{bp}\left(\mathrm{MM} 294\right.$ lac:: $\left.c a t\left[\mathrm{Cm}^{\mathrm{R}}\right]\right)$, lane 7, negative control with Cat2 and LacI5 pair of primers (MM294lac $\Delta$ cat $\left.\left[\mathrm{Cm}^{\mathrm{S}}\right]\right)$.

Linear DNA fragments were prepared as follows. Typically, plasmid DNA $(1 \mu \mathrm{g})$ was cut with ApaLI and NotI restriction endonucleases in standard conditions. The mixture was electrophoresed on a $0.7 \%$ agarose preparative gel to remove DNA fragments containing origin of replication. Appropriate DNA fragment was recovered by electroelution. The DNA pellet was suspended in TE [Tris-HCl ( $\mathrm{pH} 8$ ), 1 mM EDTA] buffer. Competent MM294 [pRecAts $\left(\mathrm{Ap}^{\mathrm{R}}\right)$ ] cells were prepared by chemical method (Miller, 1972) after IPTG(0.1 mM)mediated induction of recA expression. For transformation, linear DNA of pMK-CmlacIZ (250 ng) and $120 \mu \mathrm{l}$ of chilled cells were used $\left(10^{8}\right.$ viable cells $\left./ \mathrm{ml}\right)$. An appropriate amount of transformation mixture was plated onto selective medium (chloramphenicol and ampicillin at concentration of $12.5 \mu \mathrm{g} / \mathrm{ml}$ and $25 \mu \mathrm{g} / \mathrm{ml}$, respectively) supplemented with X-Gal/IPTG $(40 \mu \mathrm{g} / \mathrm{ml}$ and $0.1 \mathrm{mM} / \mathrm{ml}$, respectively) at $30^{\circ} \mathrm{C}$. The $\mathrm{RecA}^{+}$helper plasmid was subsequently eliminated from $\mathrm{Cm}$-positive recombinants (MM294lac:: cat $\left[\mathrm{Cm}^{\mathrm{R}}\right]$, white colonies) by increasing the temperature to $42^{\circ} \mathrm{C}$ (Wild et al., 1998, Fig. 2A). To achieve unmarked recombinants we could delete the segment carrying antibiotic resistance gene in vivo by transient expression of FLP gene from a suicidal helper plasmid pFT-Km (Posfai et al., 1997) simply by addition of autoclaved chlortetracycline $(20 \mu \mathrm{g} / \mathrm{ml})$. Flp promotes recombination at specific $13 \mathrm{bp}$ sites within 65 bp sequence, termed FRT. The resulting strain was 
named MM294lac $\Delta$ cat $\left[\mathrm{Cm}^{\mathrm{s}}\right]$. Gene disruption mutants were verified by lac phenotype analysis and tested for chloramphenicol susceptibility. Also, analytical PCR before and after cat gene excision was performed with primers specific to lacI, cat or lacZ genes in engineered region of the chromosome, as shown at Fig. 2C. In addition, the mutants were checked for the absence of any plasmids. The frequency of 4-12 gene replacements was obtained, when normalized to $1 \mu \mathrm{g}$ of DNA. Our results are in the range of recombination efficiency obtained for linear (Chi)-DNA used to target plasmid or chromosome, 2.8 gene replacements using 200-400 ng of DNA (Dabert and Smith, 1997) and 14-64 gene replacements with $1 \mu \mathrm{g}$ of DNA (Jessen et al., 1998), respectively.

Taken together we report on the construction and use of a set of integration vectors that enable introduction of any DNA fragment into the bacterial genome regardless of the actual RecA phenotype. In conjunction, Flp recombinase-based system offers opportunity for precise in vivo excision of an antibiotic resistance gene, served initially as a selection marker. Recombination between FRT sites situated on either side of the resistance determinant leads to its deletion, resulting in an unmarked mutant. Remaining FRT site can be used as a target for new exchangeable insertions.

\section{Literature}

Biek D.P. and S.N. Cohen. 1986. Identification and characterization of $r e c D$, a gene affecting plasmid maintenance and recombination in Escherichia coli. J. Bacteriol. 167: 594-603.

Bolivar F., R.L. Rodriguez, P.J. Greene, M.C. Beltach, H.L. Heyneker, H.W. Boyer, J.H. Crosa and S. Falkow. 1977. Construction and characterization of new cloning vehicles, II: a multipurpose cloning system. Gene 2: 95-113.

Cherepanov P.P. and W. Wackernagel. 1995. Gene disruption in Escherichia coli: $\mathrm{Tc}^{\mathrm{R}}$ and $\mathrm{Km}^{\mathrm{R}}$ cassettes with the option of Flpcatalyzed excision of the antibiotic-resistance determinant. Gene 158: 9-14.

Dabert P., S.D. Ehrlich and A. Gruss. 1992. $\chi$ sequence protects against RecBCD degradation of DNA in vivo. Proc. Natl. Acad. Sci. USA 89: 12073-12077.

Dabert P. and G.R. Smith. 1997. Gene replacement with linear DNA fragments in wild-type Escherichia coli: enhancement by Chi Sites. Genetics 145: 877-889.

Datsenko K.A. and B.L. Wanner. 2000. One-step inactivation of chromosomal genes in Escherichia coli K-12 using PCR products. Proc. Natl. Acad. Sci USA 97: 6640-6645.

Feher T., V. Burland and G. Posfai. 2012. In the fast line: Largescale bacterial genome engineering. J. Biotechnol. 160:72-79.

Gay N.J. 1984. Construction and characterisation of an Escherichia coli strain with a uncI mutation. J. Bacteriol. 97: 820-825.
Hamilton C.M., M. Aldea, B.K. Washburn, P. Babitzke and S.R. Kushner. 1989. New method for generating deletions and gene replacements in Escherichia coli. J. Bacteriol. 171: 4617-4622.

Jasin M. and P. Schimmel. 1984. Deletion of an essential gene in Escherichia coli by site-specific recombination with linear DNA fragments. J. Bacteriol. 159: 783-786.

Jessen J.R., A. Meng, R.J. McFarlane, B.H. Paw, L.I. Zon, G.R. Smith and S. Lin. 1998. Modification of bacterial artificial chromosomes through Chi-stimulated homologous recombination and its application in zebrafish transgenesis. Proc. Natl. Acad. Sci. USA 95: 5121-5126. Karoui El M., S.K. Amundsen, P. Dabert and A. Gruss. 1999. Gene replacement with linear DNA in electroporated wild-type Escherichia coli. Nucleic Acids Res. 27: 1296-1299.

Kim, J., C. Zwieb, C. Wu and S. Adhya. 1989. Bending of DNA by gene-regulatory proteins: construction and use of a DNA bending vector. Gene 85: 15-23.

Martinez-Morales F., A.C. Borges, A. Martinez, K.T. Shanmugam and L.O. Ingram. 1999. Chromosomal integration of heterologous DNA in Escherichia coli with precise removal of markers and replicons used during construction. J. Bacteriol. 181: 7143-7148.

Meselson M. and R. Yuan. 1968. DNA restriction enzyme from E. coli. Nature 217: 1110-1114.

Miller J.H. 1972. Experiments in Molecular Genetics. Cold Spring Harbor, NY. Cold Spring Harbor Laboratory, 352-355.

Posfai G., M. Koob, Z. Hradecna, N. Hasan, M. Filutowicz and W. Szybalski. 1994. In vivo excision and amplification of large segments of the Escherichia coli genome. Nucleic Acids Res. 22: 2392-2398.

Posfai G., M.D. Koob, H.A. Kirkpatrick and F.R. Blattner. 1997. Versatile insertion plasmids for targeted genome manipulations in bacteria: isolation, deletion, and rescue of the pathogenicity island LEE of the Escherichia coli O157:H7 genome. J. Bacteriol. 179: $4426-4428$.

Reece K.R. and G.J. Phillips. 1995. New plasmids carrying antibiotic-resistance cassettes. Gene 165: 141-142.

Roca A.I. and M.M. Cox. 1997. RecA protein: structure, function, and role in recombinational DNA repair. Prog. Nucleic Acid Res. Mol. Biol. 56: 129-223.

Sektas M. 2000. The application of homologous recombinationbased methods for genetic engineering of bacteria (in Polish). Post. Mikrobiol. 39: 199-215.

Sektas M., N. Hasan and W. Szybalski. 2001. Expression plasmid with a very tight two-step control: Int/att-mediated gene inversion with respect to the stationary promoter. Gene 267: 213-220.

Sektas, M. and W. Szybalski. 1998. Tightly controlled two-stage expression vectors employing the Flp/FRT-mediated inversion of cloned genes. Mol. Biotechnol. 9: 17-24.

Smith G.R. 2012. How RecBCD enzyme and Chi promote break repair and recombination: a molecular biologist's view. Microbiol. Mol. Biol. Rev. 76: 217-228.

Wild J., M. Sektas M., Z. Hradecna and W. Szybalski W. 1998. Targeting and retrofitting pre-existing libraries of transposon insertions with FRT and oriV elements for in-vivo generation of large quantities of any genomic fragment. Gene 223: 55-66.

Yanisch-Perron, C., J. Vieira and J. Messing. 1985. Improved M13 phage cloning vectors and host strains: nucleotide sequences of the M13mp18 and pUC19 vectors. Gene 33: 103-111. 